\title{
Exploration of Improved Methodology for Character Image Recognition of Two Popular Indian Scripts using Gabor Feature with Hidden Markov Model
}

\author{
Shubhra Saxena \\ Dept. of Computer Science \\ Jaipur National University \\ Jaipur,Rajasthan
}

\author{
V S Dhaka \\ Dept. of Computer Science \\ Jaipur National University \\ Jaipur,Rajasthan
}

\begin{abstract}
Handwritten character recognition plays an important role in the modern world. It can solve more complex problems and make the human's job easier. The present work portrays a novel approach in recognizing handwritten cursive character using Hidden Markov Model (HMM) . The method exploits the HMM formalism to capture the dynamics of input patterns, by applying a Gabor filter to a character image, observation feature vector is obtained, and used to form feature vectors for recognition. The HMM model is proposed to recognize a character image. All the experiments are conducted by using the Matlab tool kit.
\end{abstract}

\section{Keywords}

Devanagari Character Recognition, Feature Extraction, Hidden Markov Model, Gabor feature.

\section{INTRODUCTION}

Handwritten Character Recognition system has been of great benefit to society due to the tremendous advent of digital computers. The Markov model is a stochastic process which models a sequence of measurable output produced by an unknown source. The model has an additional ability to predict the future observations of the process [1]. The Markov models are most popular for temporal classification. If a model only relies on the system's statistical properties, then it is known as stochastic model [2]. It has found its application in the areas like speech, handwriting, gesture recognition and also in statistical models of real-world processes. This model assumes that the outcome or observations of the model are a probabilistic function of each state, and each state produces a number of outputs according to a unique probability distribution. Each distinct output can potentially be generated at any state. This is hidden because the state sequence is not directly observable, it can only be approximated from the sequence of observation produced by the system. The hidden Markov model has many applications, among which the most famous use is in of cursive writing into characters, prediction of rainfall on successive days. Hidden Markov Model is also used (1) to infer the most likely sequence of states that produced a given output sequence, or (2) to infer which will be the most likely next state and (3) to calculate the probability that a given sequence of outputs originated from the system.

\section{OVERVIEW OF HMM AND GABOR FILTER}

2.1. Elements of HMM

A Hidden Markov Model is a finite learnable stochastic automate. It can be summarized as a kind of double stochastic process with the two following aspects [3]:

The first stochastic process is a finite set of states, where each of them is generally associated with a multidimensional probability distribution. The transitions between the different states are statistically organized by a set of probabilities called transition probabilities.

In the second stochastic process, in any state an event can be observed. Since just analyze what is observed without seeing at which states it occurred, The states are "hidden" to the observer, therefore the name is "Hidden Markov Model".

Each Hidden Markov Model is defined by states, state probabilities, transition probabilities, emission probabilities and initial probabilities.

In order to define an HMM completely, the following five elements are to be defined [4]:

1. The $\mathrm{N}$ states of the Model, defined by

$$
\mathrm{S}=\left\{\mathrm{S}_{1}, \ldots, \mathrm{S}_{\mathrm{N}}\right\}
$$

The $\mathrm{N}$ is observation symbols per state $\mathrm{V}=\left\{\mathrm{v}_{1}, \ldots, v_{\mathrm{n}}\right\}$. If the observations are continuous then $\mathrm{N}$ is infinite.

2. The State transition probability distribution $\mathrm{A}=$ $\left\{m_{i j}\right\}$, where $m_{i j}$ is the probability that the state at time $t+1$ is $S_{j}$, is given when the state at time $t$ is $S_{i}$. The structure of this stochastic matrix defines the connection structure of the model. If a coefficient $a_{i j}$ is zero, it will remain zero even through the training process, so there will never be a transition from state $S_{\mathrm{i}}$ to $\mathrm{Sj}$.

$\mathrm{m}_{\mathrm{ij}}=\mathrm{p}\left\{\mathrm{q}_{\mathrm{t}+1}=\mathrm{j} \mid \mathrm{q}_{\mathrm{t}}=\mathrm{i}\right\}, \quad \mathrm{I} \leq \mathrm{i}, \mathrm{j} \leq \mathrm{N}$

Where $\mathrm{q}_{\mathrm{t}}$ denotes the current state. The transition probabilities should satisfy the normal stochastic constraints.

$a_{i j} \geq 0,1 \leq \mathrm{i}, \mathrm{j} \leq \mathrm{N}$ and $\sum_{\mathrm{j}=1}^{\mathrm{N}} \mathrm{a}_{\mathrm{ij}}=1,1 \leq \mathrm{i} \leq N$. 
3. The observation symbol probability distribution in each state, $\left.\left.N=\left\{n_{j}\right) k\right)\right\}$ where $n_{j}(k)$ is the probability that symbol $v_{\mathrm{k}}$ is emitted in state $\mathrm{S}_{\mathrm{j}}$,

$n_{j}(k)=j p\left\{u_{t}=u_{k} \mid q_{t}=j\right\}, 1 \leq j \leq N, \quad 1 \leq k \leq M$

Where $v_{\mathrm{k}}$ denotes the $\mathrm{k}^{\text {th }}$ observation symbol in the alphabet, and $v_{\mathrm{t}}$ is the current parameter vector.

The following stochastic constraints must be satisfied:

$$
n_{j}(k) \geq 0,1 \leq \mathrm{j} \leq \mathrm{N}, 1 \leq k \leq M \text { and } \sum_{\mathrm{k}=1}^{\mathrm{M}} \mathrm{n}_{\mathrm{j}}(k)=1,1 \leq \mathrm{j} \leq N
$$

4. If the observations are continuous, then the continuous probability density function is used, instead of a set of discrete probabilities. Usually the probability density is approximated by a weighted sum of M Gaussian distribution N,

$$
b_{j}\left(0_{t}\right) \sum_{\mathrm{m}=1}^{\mathrm{M}} \mathrm{c}_{\mathrm{jm}} N\left(\mu_{j m}, \sum s_{\mathrm{jm}}, 0_{t}\right)
$$

where $c_{j m}=$ weighting coefficients, $\mu_{\varphi m}=$ mean vectors, and $\quad \mathrm{sjm}=$ Covariance matrices, $\mathrm{c}_{\mathrm{jm}}$ should also satisfy the stochastic assumptions $\mathrm{c}_{\mathrm{jm}} \geq 0,1 \leq \mathrm{j} \leq \mathrm{N}, 1 \leq \mathrm{m} \leq \mathrm{M}$ and

$$
\sum_{\mathrm{m}=1}^{\mathrm{M}} \mathrm{c}_{\mathrm{jm}}=1,1 \leq \mathrm{j} \leq \mathrm{N}
$$

5. The HMM is the initial state distribution $\pi=\left\{\pi_{\mathrm{i}}\right\}$, where $\pi_{\mathrm{i}}$ is the probability that the model is in state $\mathrm{Si}$ at the time $\mathrm{t}=0$ with

$\pi_{\mathrm{i}}=\mathrm{p}\left\{\mathrm{q}_{1}=1\right.$ and $1 \leq \mathrm{i} \leq \mathrm{N}$

\subsection{Gabor Filter}

A Gabor filter is a linear filter whose impulse response is defined by a harmonic function multiplied by a Gaussian function [6]. Gabor filters are directly related to Gabor wavelets, since they can be designed for a number of dilations and rotations. However, in general, expansion is not applied for Gabor wavelets, since this requires computation of bi orthogonal wavelets, which may be very time-consuming. Therefore, a filter bank consisting of Gabor filters with various scales and rotations is created.The filters are convolved with the signal, resulting is called Gabor space. Gabor space is very useful in, e.g., Image processing applications such as iris recognition and fingerprint recognition.

Gabor Filter has been used in many applications, such as texture segmentation, target detection, fractal dimension management, document analysis, edge detection, retina identification, image coding and image representation [7]. A Gabor filter can be viewed as a sinusoidal plane or particular frequency and orientation, modulated by a Gaussian envelope [8] -[9].

\subsubsection{Properties of Gabor Filter}

- Simple Gabor Feature Space

Different Gabor filter parameters can be used to extract different features. The frequency and orientation of the Gabor filters are some of the most useful parameters. In order to distinguish between objects in most cases it is necessary to apply several Gabor filters to an image, by forming a so called 'filter bank', and then for classification purposes the relationship between Gabor responses of different images is calculated.The selection of discrete rotation angle $\theta_{\mathrm{k}}$ is very important, and it has been shown the orientation must be spaced uniformly as [10]:

$$
\theta_{k}=\frac{k 2 \pi}{n} \quad \text { for } k=0, \ldots, n-1
$$

where $\theta_{\mathrm{k}}$ is the $\mathrm{k}^{\text {th }}$ orientation and $\mathrm{n}$ is the number of orientations to be used. However, in this paper the computation is reduced to half, as for real signals the responses on angles $(\pi, 2 \pi)$ are $90^{\circ}$ phase shifted from responses on $(0, \pi)[11]$ :

$$
\theta_{\mathrm{k}}=\frac{\mathrm{k} \pi}{\mathrm{n}} \text { for } \mathrm{k}=0, \ldots, \mathrm{n}-1
$$

This equation represents the scale invariance property of the Gabor filters, but in real applications, it is required to establish spacing between the scales. In order to maintain homogenous spacing between the scales an algorithmic relation between the frequencies $\mathrm{f}$ has been established:

$\mathrm{F}_{\mathrm{k}}=\mathrm{a}^{-\mathrm{k}} \mathrm{f}_{\max }, \quad$ for $\mathrm{k}=0, \ldots, \mathrm{n}-1$

where $f_{k}$ is the kth frequency, $f_{0}=f_{\max }$ is the highest frequency desired, and $\mathrm{a}$ is the frequency scaling factor. In this work a scaling factor of $\sqrt{2}$ is used which has been used for halfoctave spacing, but another useful value for octave spacing is $\mathrm{a}=2$.

\section{METHODOLOGY}

The first stage is an Image pre-processing stage in which data in a paper document are captured by optical scanner, then pixel based images created by painting programs such as Microsoft's Paintbrush. These pixels may have values: OFF (0) or ON (1) for binary images, 0-255 for gray-scale images, and 3 channels of 0-255 color values for color images.

The images are read into the Matlab environment using imread function. Here filename is a string containing the complete name of the image file and then the image can be displayed on the MatLab workspace using function imshow().

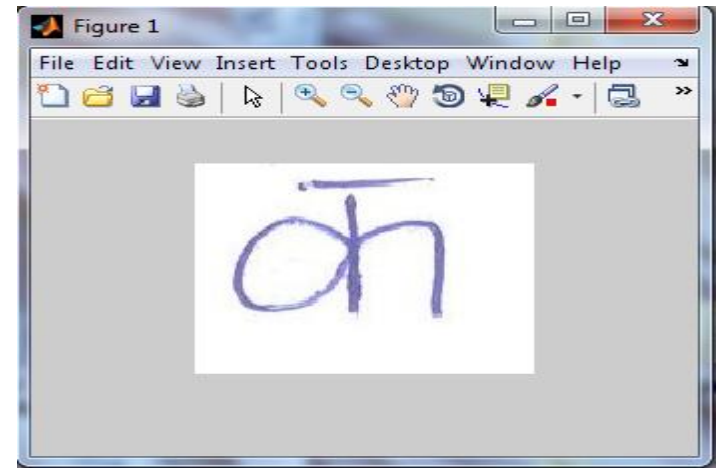

Fig.1 Sample Image of Character क

The logical function is used to convert gray image to logical. It returns an array that is further used to perform 
logical indexing Logical can have the values 0 and 1. After that $\operatorname{Im} 2 b w$ function of Matlab is used to convert images to binary image from indexing image.

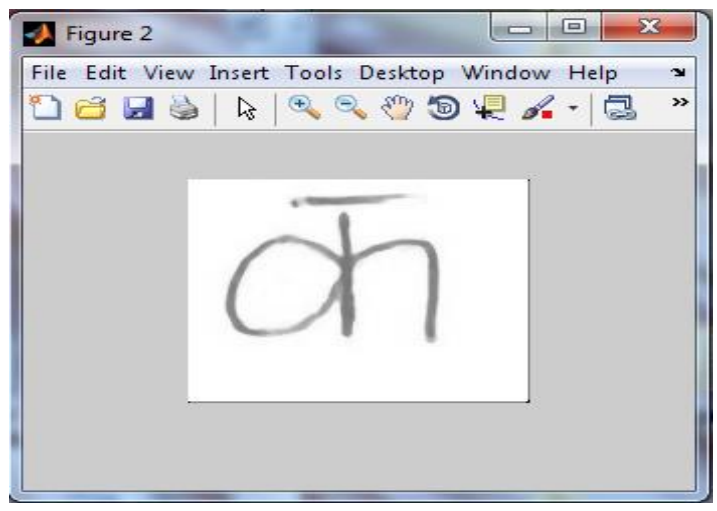

Fig.2 Gray Scaled Image of Character क

In the next stage image is enhanced using median filter image processing function. This pre-processing stage yields a clean document with minimum noise.

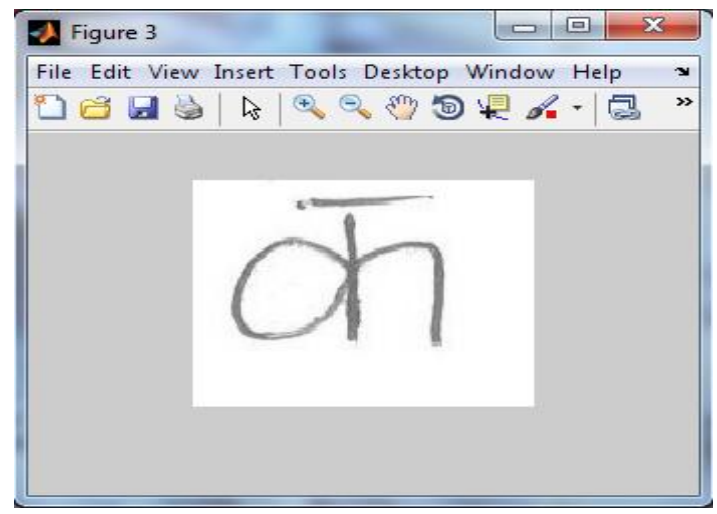

Fig.3 Noise removal Image of Character क

\subsection{Gabor Filter Approach}

The next phase of identification is a feature extraction phase. Different Gabor filter parameters can be used to extract different features. The frequency and orientation of the Gabor filters are just some of the most useful parameters. In order to distinguish between patterns it is necessary to apply several Gabor filters to an image, by forming a so called 'filter bank', and then for extracting feature purpose the Gabor responses of different character images have been calculated. The orientation angle of this set of Gabor filter is given by [12]. In this case the character is divided into overlapping horizontal strips of height $h$ pixels where the strips overlap by $\mathrm{p}$ pixel. Each horizontal strip is subsequently segmented vertically into blocks of $\mathrm{k}$ pixels, with overlap of pixels. For an image with width $\mathrm{w}$ and height $\mathrm{h}$, there will be approximately $((\mathrm{h} / \mathrm{j}-$ p)) +1$) *(w /(k-p))$ blocks. Each character image, then undergoes wavelet decomposition, producing an average image and a sequence of detailed image. For the character images, 3-level wavelet decomposition is employed, producing a vector with one average image and fifteen detail images. The L2 form of the image is calculated that is further used to from the observation vector for those blocks. The L2 norms of an image are the square root of the sum of all pixel values squared.

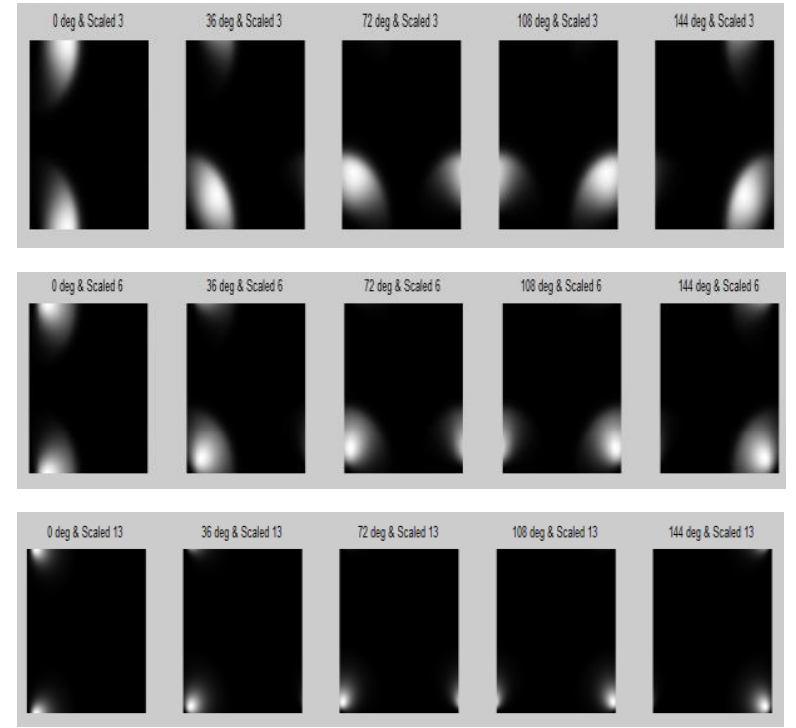

Fig4. Log Gabor Filter Images of Character क

The character image is convoluted with a number of Gabor filters with 5orientation and 3 scales being used. The output image is split into blocks on each block DWT is calculated. For each block, L2 norm is calculated. Therefore, each block from the original image can be represented by a feature vector of ( 5 orientation $\times 3$ scales) [14].
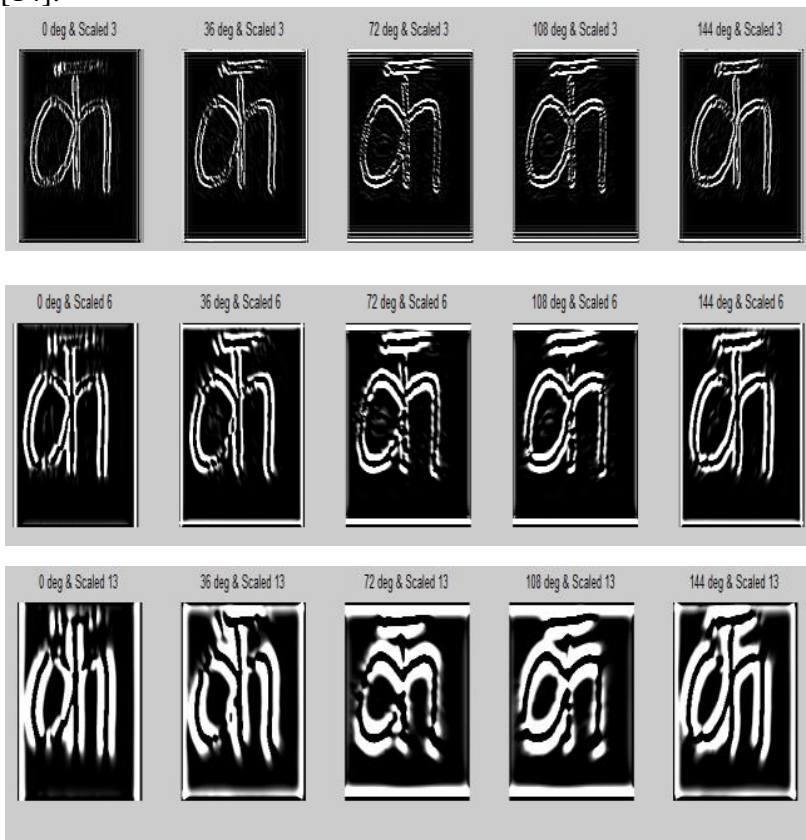

Fig.5.Response Matrix Images ( क From which feature is extracted)

\subsection{Hidden Markov Model Approach}

The image observation vector is calculated and the feature is collected from all image blocks, from horizontally and vertically.

Each shape represents a random variable that can adopt any of a number of values. The random variable $s(t)$ is the hidden state at time $\mathrm{t}$. The random variable $\mathrm{O}(\mathrm{t})$ was observed at the time. 
By the second stochastic process, the value of the observed variable $\mathrm{O}(\mathrm{t})$ depends on the value of the hidden variable $\mathrm{s}(\mathrm{t})$ also at the time $\mathrm{t}$.

Hidden Markov Model with multivariate Gaussian state conditional distribution consists of

$\mathrm{P}_{\mathrm{o}}(\mathrm{i})=$ Row vector containing the probability distribution of the model $\lambda$ being in the state $s_{i}$ at time $t$ when presented with the sequence of observation $\mathrm{O}$,

$$
\mathrm{PO}(\mathrm{i})=\mathrm{P}(\text { is at } \mathrm{t}(\mathrm{O}, \lambda)
$$

$\mathrm{T}=$ Transition Matrix containing the probability of the model $\lambda$ being in the state $s_{i}$ at time $t$ and making a transition to state $s_{j}$ at time $t+1$ when presented with the sequence of observation O.

$$
a(i, j)=P\left(s_{i} \text { at } t, s_{j} \text { at } t+1 \mid 0, \lambda\right)
$$

For the purpose of handwritten character recognition left right model is considered. In left to right model transition from state $i$ to state $j$ is only allowed if $j>=i$, resulting in a smaller number of transition probabilities is to be learned. The clusters of observation are created separately by estimating feature set generated by Gabor filter.

For transition matrix $\mathrm{T}$, the row vector summation must be equal to 1 for any number of states.

Table 1. Transition Matrix

\begin{tabular}{|l|l|l|l|l|}
\hline 0.8 & 0.2 & 0.0 & 0.0 & 0.0 \\
\hline 0.0 & 0.0 & 0.8 & 0.2 & 0.0 \\
\hline 0.0 & 0.8 & 0.2 & 0.0 & 0.0 \\
\hline 0.2 & 0.0 & 0.0 & 0.0 & 0.8 \\
\hline 0.0 & 0.0 & 0.0 & 0.8 & 0.2 \\
\hline
\end{tabular}

The vector, along with the observation vector from all other training images of the same individual, is used to train the Hidden Markov Model for this individual using Baum -Welch algorithm [6], [13]. One hidden markov model is used for each block of character image to test the accuracy.

Table 2. Feature Set of Character क

\begin{tabular}{|l|l|l|l|l|}
\hline \multicolumn{5}{|c|}{ Feature Set 1(gabor SquareEnergy) } \\
\hline 0.55 & 1.25 & 2.23 & 0.17 & 0.49 \\
\hline 0.93 & 0.39 & 0.68 & 0.94 & 0.40 \\
\hline 0.78 & 1.13 & 0.20 & 0.62 & 1.17 \\
\hline \multicolumn{5}{|l|}{} \\
\hline \multicolumn{5}{|l|}{ Feature Set 2(gabor MeanAmplitude) } \\
\hline 1.67 & 3.14 & 4.89 & 1.16 & 2.16 \\
\hline 3.36 & 1.37 & 2.23 & 3.24 & 1.45 \\
\hline 2.48 & 3.66 & 1.28 & 2.49 & 3.90 \\
\end{tabular}

\section{EXPERIMENTS AND RESULTS}

\subsection{Analysis}

Based on the observation feature vector extracted from all the training samples in a class, the parameters of the corresponding Hidden Markov model are estimated using maximum likelihood algorithm. An unknown word image having an observation sequence $\overrightarrow{0}=\mathrm{O}_{1}, \ldots, \mathrm{O}_{\mathrm{T}}$ is classified in the character class for which the probability is maximum. In other words, the unknown image is classified in class $\mathrm{c}$.

$$
c=\operatorname{maxg}_{1 \leq j \leq m} p\left(\frac{\overrightarrow{0}}{\lambda_{j}}\right)
$$

For a classifier of $\mathrm{m}$ clases of samples, $\mathrm{m}$ denote different Hidden Markov models by $\lambda_{\mathrm{j}}, \mathrm{j}=1, \ldots, \mathrm{m}$. Let an input sample $\mathrm{B}$ of an unknown class have an observation sequence $\bar{o}$. For each trained model, The likelihood of that Hidden markov model producing the observation vector is calculated. The probability ${ }_{P}\left(\frac{\bar{o}}{\lambda \mathrm{j}}\right)$ is computed for each model $\lambda_{\mathrm{j}}$ and $\mathrm{B}$ is assigned to class $\mathrm{c}$ whose model shows the highest probability.

\subsection{Testing}

In order to maintain the identity of an image, a feature vector for that image is used to train the system. For each trained Model, the likelihood of that Hidden Markov model producing the observation vector is calculated. As the identification, process assumes that all sample belongs to known individuals, the image is recognized that have the highest likelihood value.

Following are the main result of handwritten character

\begin{tabular}{|c|c|c|c|c|}
\hline $\begin{array}{l}\text { Charact } \\
\text { er }\end{array}$ & $\begin{array}{l}\text { Confused } \\
\text { Recogniz } \\
\text { ed } \\
\text { Character } \\
\text { s }\end{array}$ & $\begin{array}{l}\text { Characte } \\
\text { rs for } \\
\text { training }\end{array}$ & $\begin{array}{l}\text { Characte } \\
\text { rs for } \\
\text { testing }\end{array}$ & $\begin{array}{l}\text { \% Characte } \\
\text { rs } \\
\text { Recognitio } \\
\text { n } \\
\text { Accuracy }\end{array}$ \\
\hline क & & 20 & 10 & $80 \%$ \\
\hline ख & & 20 & 10 & $88 \%$ \\
\hline ग & म & 20 & 10 & $70 \%$ \\
\hline घ & है & 20 & 10 & $61 \%$ \\
\hline ड & & 10 & 10 & $68 \%$ \\
\hline च & & 20 & 5 & $85 \%$ \\
\hline 8 & & 20 & 5 & $71 \%$ \\
\hline ज & ज & 10 & 5 & $65 \%$ \\
\hline झ & & 20 & 5 & $82 \%$ \\
\hline
\end{tabular}
recognition:-

Table 3. Feature Set of Character 


\begin{tabular}{|c|c|c|c|c|}
\hline ज & ज & 10 & 6 & $65 \%$ \\
\hline ट & उ द & 10 & 5 & $69 \%$ \\
\hline $\bar{\delta}$ & & 20 & 4 & $86 \%$ \\
\hline ड & & 20 & 4 & $89 \%$ \\
\hline ढ & ट & 10 & 7 & $65 \%$ \\
\hline ण & & 20 & 4 & $80 \%$ \\
\hline त & & 20 & 5 & $85 \%$ \\
\hline थ & & 20 & 5 & $75 \%$ \\
\hline द & & 20 & 5 & $85 \%$ \\
\hline $\bar{घ}$ & घ & 10 & 5 & $61 \%$ \\
\hline न & & 20 & 5 & $88 \%$ \\
\hline प & य & 10 & 5 & $67 \%$ \\
\hline $\bar{\phi}$ & & 20 & 5 & $84 \%$ \\
\hline ब्্ & व & 10 & 5 & $61 \%$ \\
\hline भ & म & 10 & 5 & $64 \%$ \\
\hline म & भा & 10 & 5 & $65 \%$ \\
\hline य & प & 10 & 5 & $67 \%$ \\
\hline $\bar{\gamma}$ & & 10 & 5 & $88 \%$ \\
\hline ल & & 10 & 6 & $72 \%$ \\
\hline व & ब্র & 10 & 4 & $61 \%$ \\
\hline श & & 10 & 5 & $70 \%$ \\
\hline ष्म & & 10 & 5 & $80 \%$ \\
\hline स & & 10 & 5 & $70 \%$ \\
\hline $\bar{\varepsilon}$ & & 10 & 5 & $89 \%$ \\
\hline क & & 10 & 5 & $80 \%$ \\
\hline$\zeta$ & & 10 & 5 & $82 \%$ \\
\hline
\end{tabular}

\subsection{Result Graph of Proposed Algorithm}

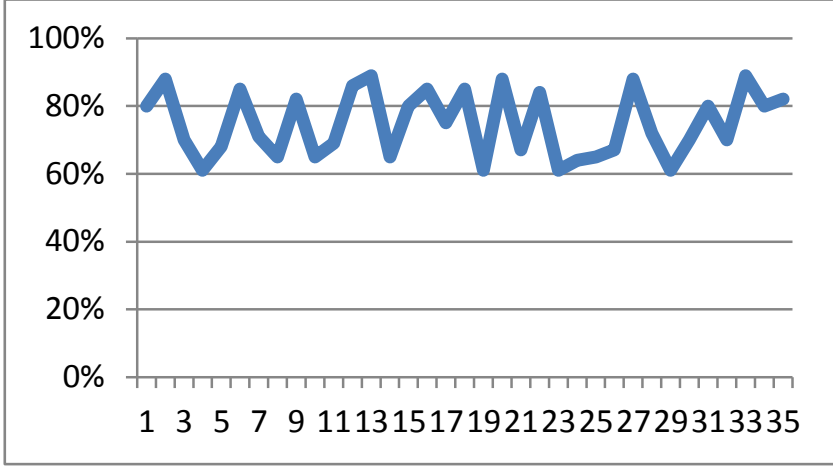

Fig.6. Result Graph of Proposed Algorithm

\section{CONCLUSION}

In this research work a new approach has been applied to recognize the Devanagari and Bangla character.

After the complete research the following points are derived:

a) Thirty five handwritten characters have been selected for this work.

b) Five hundred Samples from these characters have been collected.

c) Each part is converted into equivalent Binary codes by using Binarization method of Matlab.

d) Gabor Filter is used for extracting the feature from the character image. Hidden Markov Model is used for training and testing the character image.

e) Training of each sample is applied up to 5 times to minimize the error.

f) Two hundred seventy characters have been applied in the network to obtain the result.

g) Based on the observation feature vector extracted from all the training samples in a class, the parameters of the corresponding hidden markov model are estimated using the maximum likelihood algorithm.

h) An unknown character image having an observation sequence $=\mathrm{O}_{1}, \ldots, \mathrm{O}_{\mathrm{T}}$ is classified in the character class for which the probability $P\left(\frac{\bar{o}}{\lambda \mathrm{j}}\right)$ is maximum.

i) Network correctly recognizes 168 samples. Therefore, the accuracy level of the network is around $84 \%$.

The proposed approach has been able to recognize a handwritten Devanagari and Bangla characters. The results of this approach are promising.The only limitation of this work is the number of Bangla characters which have been selected for the work. The future work is to select all Bangla character for recognition through this approach.

\section{REFERENCES}

[1] W. Khreicha,E. Granger, A.Miri , R.Sabourina, "A survey of techniques for incremental learning of HMM parameters",Journal of Information Sciences, vol.197,pp.105-130,2012.

[2] J. Nielsen,A. Sand, "Algorithms for a parallel implementation of Hidden Markov Model with a state 
space", IEEE transaction on Parallel and distributed Processing Symposium, pp.447-454,2011.

[3] Shai Fine,Yoram Singr,Naf Tali Tishbay, "The Hierarichal Hidden Markov Model: Analysis and its Applications", Kluwer Academic Publisher, Boston, pp.41-62,1998.

[4] N.Africa, F.Yarman, "HMM Based Handwritten Recognition”, Proceeding of ISCIS , pp.260-266,1997.

[5] J. R. Movellan "Tutorial on Gabor Filter" GNU Documentation [Free License, pp.1-20, 2002.

[6] Li Ying-Chun, Li Zhan-Chun, Y. Mei, J. Zhang, "Detecting algorithms based Gabor in Microscopic image", Proceedings of the Fourth International Conference on Machine Learning and Cybernetics, Guangzhou, pp. 5410-5415, 2005.

[7] A. Krishnan, "Evaluation of Gabor Filter Parameters for image Enhancement and Segmentation", M.Tech. Thesis , 2009.

[8] S. Berisha, "Image Classification using Gabor Filter and Machine Learning ", M.Tech Thesis, Wake Forest University, 2009.

[9] L. R. Bahl, F. Jelinek and L. R. Mercer, A Maximum Likelihood Approach to Continuous Speech Recognition, IEEE Transaction on Pattern Analysis and Machine Intelligence, pp.179-190, 1983.
[10] A. Krishnan, "Evaluation of Gabor Filter Parameters for image Enhancement and Segmentation" M.Tech. Thesis, 2009.

[11] M. Wagn, Q. Han, Y. Tu, G. Chen, Y. Gao, "Unsuperivsed Texture Image segmentation Based on Gabor Wavelet and multi-PCNN", School of Computer Science and Engineering, South Chine University of Technology, China, vol.2, pp. 376-381, 2008.

[12] Wei, M. Bartels, "Unsupervised segmentation Using Gabor wavelets and statistical features in LIDAR Data Analysis" Proceedings of the $18^{\text {th }}$ International Conference on Pattern Recognition, vol.1, pp.667-670, 2005.

[13] G.G Rajput , Anita H.B “ Handwritten Script Recognition using DCT and Wavelet Features at Block Level”, International Journal of Computer Application , Recent Trends in image processing and pattern recognition,pp.158-163,2010.

[14] B.Shaw,S. K. Parui, M. Shridhar, "Offline handwritten Devanagari word recognition : A holistic approach based on directional chain code feature and HMM", IEEE International Conference on information techonology,pp.203-208, 2008.

[15] P.Mukherji, P. Rege, "Shape Feature and Fuzzy Logic Based Offline Devanagari Handwritten Optical Character Recognition", International Journal of Pattern Recognition Research, pp.52-68, 2009. 